\title{
Graphical Frobenius representations
}

\author{
John Kevin Doyle ${ }^{1}$ - Thomas W. Tucker ${ }^{2}$ - Mark E. Watkins ${ }^{3}$
}

Received: 9 December 2016 / Accepted: 30 January 2018 / Published online: 6 February 2018

C Springer Science+Business Media, LLC, part of Springer Nature 2018

\begin{abstract}
A Frobenius group is a transitive permutation group that is not regular and such that only the identity fixes more than one point. A graphical Frobenius representation $(G F R)$ of a Frobenius group $G$ is a graph whose automorphism group, as a group of permutations of the vertex set, is isomorphic to $G$. The problem of classifying which Frobenius groups admit a GFR is a natural extension of the classification of groups that have a graphical regular representation (GRR), which occupied many authors from 1958 through 1982. In this paper, we review for graph theorists some standard and deep results about finite Frobenius groups, determine classes of finite Frobenius groups and individual groups that do and do not admit GFRs, and classify those Frobenius groups of order at most 300 having a GFR. Because a Frobenius group, as opposed to a regular permutation group, has a highly restricted structure, the GFR problem emerges as algebraically more complex than the GRR problem. This paper concludes with some further questions and a strong conjecture.
\end{abstract}

Keywords Frobenius group · Cayley graph · Automorphism · Graphical regular representation

John Kevin Doyle: Deceased 21 June, 2012.

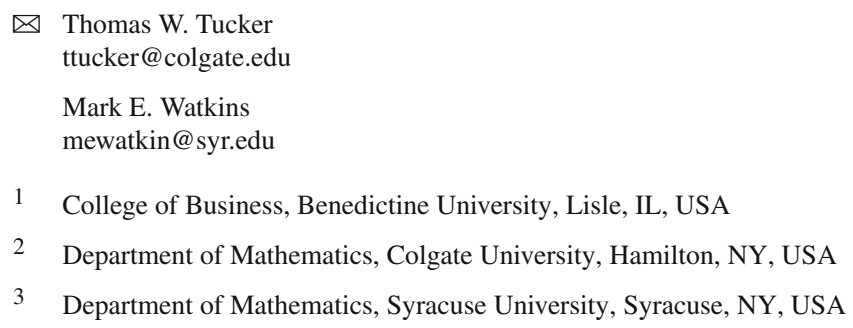




\section{Introduction and motivation}

The present work furthers the inquiry as to how small the automorphism group $\operatorname{Aut}(\Gamma)$ of a finite graph $\Gamma$ can be, perhaps subject to certain conditions, but without sacrificing transitivity. Clearly the minimal situation is when its vertex stabilizers $\operatorname{Aut}_{x}(\Gamma)$ for $x \in V \Gamma$ are trivial. In this case, Aut $(\Gamma)$ is regular (transitive and every nonidentity element is fixed-point free), and hence, $\Gamma$ must be a Cayley graph of $G$ (see [28]). That is, there is an inverse closed generating set $S$ of $G$ such that the vertex set of $\Gamma$ is $G$ and for distinct $x, y \in G$, the 2-set $\{x, y\}$ is an edge if and only if $y=x s$ for some $s \in S$. We then write $\Gamma=\operatorname{Cay}(G, S)$, and we say that $\Gamma$ is a graphical regular representation $(G R R)$ of the (abstract) group $G$. In the context of Cayley graphs, the set $S$ is usually called the connection set.

The question as to which abstract groups admit a GRR has been known as the GRR problem. A number of graph theorists ${ }^{1}$ have contributed to its complete solution. Here is a detailed characterization for finitely generated groups.

Theorem 1.1 A finitely generated group $G$ admits a GRR if and only if $G$ is not one of the following:

(1) an Abelian group that is not an elementary Abelian 2-group;

(2) a generalized dicyclic group;

(3) $\left(\mathbb{Z}_{2}\right)^{m}$ for $m=2,3$, 4 or any of the following ten non-Abelian groups: the dihedral groups $D_{3}, D_{4}, D_{5}$; the alternating group Alt(4); two groups of order 16 (one of exponent 4, one of exponent 8); the group of order 18 with exponent 6; the group of order 27 with exponent 3 ; the groups $\mathbb{Z}_{3} \times Q$ and $\mathbb{Z}_{4} \times Q$.

An Abelian group is an elementary Abelian p-group, for prime $p$, if it has exponent $p$. A group $G$ is generalized dicyclic if it contains an Abelian subgroup $A$ of index 2 that is not an elementary Abelian 2-group and an element $y \in G \backslash A$ of order 4 such that $y^{-1} x y=x^{-1}$ for all $x \in A$; when $A$ is cyclic, $G$ is called dicyclic. The quaternion group $Q$ (of order 8 ) is the smallest dicyclic group. For each $m \geq 2$, there is exactly one dicyclic group of order $4 m$, which is called a generalized quaternion when $m=2^{e}$. Each member of the infinite families (1) and (2) in Theorem 1.1 admits an involutory automorphism that takes each element to itself or its inverse, thus providing a nontrivial group automorphism that leaves invariant any inverse closed connection set. No other groups have such an automorphism. The final classification of GRRs says that, beyond some "low-level noise," the groups having no GRR fail to do so for this very reason.

For regular permutation groups only the identity fixes a point. The natural next choice is a Frobenius group: a permutation group that is transitive but not regular and only the identity fixes more than one point. In analogy to the GRR problem, we inquire in the present paper which Frobenius groups $G$ have a graphical Frobenius representation $(G F R)$, that is, a graph whose automorphism group as a permutation

\footnotetext{
1 Main contributors to the solution of the GRR problem include (chronologically with some overlap) G. Sabidussi, W.Imrich, M.E. Watkins, L. A. Nowitz, D. Hetzel, C. D. Godsil, and L. Babai. See [2, 12,14$16,19,26,29,32-35]$ and [36].
} 
group on its vertex set is the same as $G$ ? We call this the GFR problem. It was the subject of the first author's Ph.D. thesis [10] and is discussed briefly in [13].

Other variations on the GRR problem may be pursued either by replacing properties of the group action (as we have done here) or by replacing the combinatorial objects upon which a regular permutation group is acting (as in the spirit of $[1,2]$ ). One can replace graphs by digraphs (the DRR problem), or one can use oriented graphs, that is, digraphs without parallel edges (the ORR problem). For recent work on these problems, see $[3,8,9,23,24]$.

Although Theorem 1.1 also embraces infinite groups, in this paper we consider the GFR problem only for finite Frobenius groups, as many fundamental group-theoretical results do not necessarily hold for infinite Frobenius groups (cf. [5,7]). (For examples of infinite GFRs, see [37].) Henceforth, all groups in this paper are finite, unless otherwise stated.

Counterintuitively perhaps, the GFR problem is largely not analogous to the GRR problem. All regular permutation group representations of an abstract group are equivalent to left or right multiplication of the group acting upon itself (depending on whether one views permutations as acting on the left or right). On the other hand, a Frobenius permutation group has a highly restricted algebraic structure, and many large classes of abstract groups do not admit Frobenius group representations. Thus, the GFR problem becomes in effect a two-stage problem:

1. Given an abstract group $G$, is it (abstractly) isomorphic to a Frobenius permutation group (on a set)?

2. Given a Frobenius permutation group $G$ on a set $V$, does there exist a graph $\Gamma$ with vertex set $V$ such that $\operatorname{Aut}(\Gamma)$ is isomorphic (as a permutation group) to $G$ ?

There exists much work in the literature of group theory regarding Question 1. In Sect. 2 of this paper we summarize the portions of this theory that we need in our pursuit of Question 2.

Section 3 draws a sharp distinction between the GRR problem and the GFR problem from the point of view of graph products; GRRs are generally built via graph products, while GFRs never are. This negative property of GFRs provides a necessary graphical condition for the automorphism group of a graph to be a Frobenius permutation group acting on its vertex set, i.e., for a graph to be a GFR.

Section 4, consisting of five subsections, is the theoretical core of this work. We prove the existence of GFRs for several infinite families of groups and characterize some infinite families of groups that do not admit a GFR. Aided by these tools, in Sect. 5 we determine all groups of order up through 300, excepting order 192, that admit/do not admit a GFR.

In Sect. 6 we pose some questions that probe beyond the results presented in this article and offer a strong conjecture.

We conclude this introduction by expressing our deep gratitude to Marston Conder, who provided us assistance at numerous points in this paper. He shared with us many MAGMA [4] computations, of which the remarks in Sect. 5 on groups of order 1029 and 192 are but two examples. He led us through much of the theory on Frobenius groups given in Sect. 2. He also pointed out to us the importance of Li's Theorem [22] in obtaining GFR's for extensions of cyclic groups. 


\section{Group-theoretical prerequisites}

The techniques that had been applied in the solution of the GRR problem were mostly graph-theoretical, as the requisite group theory extended barely beyond the notion of solvability. In contrast, the GFR problem requires a much greater knowledge of the theory of permutation groups in general and of Frobenius groups in particular. Indeed, the theory of Frobenius groups involves some of the deepest and most beautiful results in group theory. Therefore, for the sake of many graph theorists (and perhaps even for some algebraists?), we devote this section to a brief summary of the essentials of the theory of Frobenius groups. We have included proofs when they are short, elementary, and instructive.

Let $X$ be a nonempty set and let $G$ be a subgroup of the symmetric group $\operatorname{Sym}(X)$. The identity permutation of $G$ is denoted by $i d$. The permutation group $G$ is transitive when, given any $x, y \in X$, there exists $\varphi \in G$ such that $\varphi(x)=y$. The stabilizer of a point $x \in X$ is the subgroup $G_{x}=\{\varphi \in G: \varphi(x)=x\}$. We say that $G$ is semiregular if $G_{x}$ is trivial for all $x \in X$, in which case all orbits of $G$ in $X$ are the same size. A permutation group is regular if it is both transitive and semiregular, in which case $|G|=|X|$, or equivalently, for all $x, y \in X$, there exists a unique permutation $\varphi \in G$ such that $\varphi(x)=y$. When $G$ is transitive, all of its stabilizers are conjugate and hence isomorphic.

Definition The Frobenius kernel, or more briefly, the kernel $K$ of a Frobenius group $G$ is the set of permutations in $G$ having no fixed point together with the identity permutation. The point stabilizer $H$ is called the complement.

It follows immediately from this definition that if the Frobenius group $G$ is acting on a set $X$ and if $H$ fixes $x$ for some $x \in X$, then $H$ acts semiregularly on $X \backslash\{x\}$. As the orbits of $H$ on $X \backslash\{x\}$ all have size $|H|$, it follows that $|H|$ divides $|X|-1$. Since conjugates of nonidentity elements of $K$ must also have no fixed point, $K$ is a normal subset of $G$. The set $K \backslash\{i d\}$ is the complement in $G$ of the union of all the conjugates of $H$. That union therefore has size $|G|-(|K|-1)$, where $|G|=|X||H|$ as $G$ is transitive. Since the intersection of any two distinct conjugates of $H$ is $\{i d\}$, that union also has size $|X||H|-(|X|-1)$. Therefore, $|K|=|X|$.

We summarize these observations as follows:

Proposition 2.1 Suppose that $K$ is the kernel of the Frobenius group $G$ acting on a set $X$. Then

(a) $|H|$ divides $|K|-1$, and so $|H|$ and $|K|$ are relatively prime;

(b) $g \mathrm{Kg}^{-1}=K$ for all $g \in G$;

(c) $|K|=|X|$.

One might hope that the kernel $K$ is a subgroup of $G$, namely that the product of two permutations in $G$ fixing no points also fixes no points. One of the first triumphs of character theory was Frobenius' proof in 1901 that $K$ is indeed a subgroup. Surprisingly, to this day, every known proof of this fact involves character theory in some form.

Theorem 2.2 (Frobenius [11]) The kernel of a Frobenius group is a subgroup of $G$, which by Proposition 2.1(b) is a normal subgroup. 
Corollary 2.3 If $G$ is a Frobenius group acting on $X$, then $G=H K$ and $K$ acts regularly on $X$.

Proof Let $x \in X$. Then $H$ or some conjugate of $H$ fixes $x$. Suppose that $k_{1}(x)=k_{2}(x)$ for some $k_{1}, k_{2} \in K$. Then $k_{2}^{-1} k_{1}$ fixes $x$. Since $K$ is a group acting semiregularly on $X$, we have $k_{2}^{-1} k_{1} \in H \cap K=\{i d\}$, that is, $k_{1}=k_{2}$. Thus, the mapping from $K$ to $X$ given by $k \mapsto k(x)$ is injective. By Proposition 2.1(c), the mapping is surjective, and so $K$ acts transitively and hence regularly on $X$. The same argument can be used to show that the mapping $k \mapsto H k$ is bijective, that is, the number of $H$-cosets is exactly $|K|=|X|$. Hence, the union of the cosets $H k$ for $k \in K$ contains all of $G$.

Since the kernel of a Frobenius group $G$ acting on an $n$-set is transitive while the complement is semiregular, it follows that $|G| \leq n(n-1)$. The smallest Frobenius groups are the symmetric group $\operatorname{Sym}(3)$ on a 3-set and the alternating group Alt(4) on a 4-set, and in these instances, $|G|=n(n-1)$ holds.

Since the kernel of a Frobenius group is a normal subgroup, there exists a purely group-theoretical definition of a Frobenius group $G$ without reference to permutation groups: $a$ Frobenius group is a finite semidirect product $K \rtimes H$ such that the action of $H$ as a subgroup of $\operatorname{Aut}(K)$ on $K \backslash\{i d\}$ is semiregular. For this alternative definition, a given abstract finite group $G$ is a Frobenius group in at most one way, since $K$ must be the Fitting subgroup of $G$ (cf. [27], p. 92). On the other hand, since a complement $H$ may be injected into $\operatorname{Aut}(K)$ in different ways, there can be more than one Frobenius group $G$ with given kernel $K$ and complement $H$. For example, if $K$ is the vector space of dimension 2 over the field $G F(5)$, the group $H=C_{4}$ can act semiregularly on $K$ either as a "rotation" of order 4 or as scalar multiplication. This gives two different Frobenius groups with the same kernel $K$ and complement $H$. In Sect. 5, we often fix $K$ and $H$ and write simply $G=H K$, but we must consider all subgroups of $\operatorname{Aut}(K)$ isomorphic to $H$ that act semiregularly on $K \backslash\{i d\}$.

Notation As we have done thus far, when speaking of a Frobenius group, we continue (but now without explicit mention) to denote its kernel by $K$ and its complement by $H$, and we write $G=H K$. We let $\operatorname{Syl}_{p}(K)$ denote the Sylow $p$-subgroup of $K$. The symbols $C_{n}$ and $D_{n}$ denote, respectively, the cyclic group of order $n$ and the dihedral group of order $2 n$. If $A$ is any group, the symbol $\alpha$ will always denote the involutory function $\alpha: x \mapsto x^{-1}$ for all $x \in A$. The group $A$ in question will be clear from the context. It is elementary that $\alpha \in \operatorname{Aut}(A)$ if and only if $A$ is an Abelian group.

The semiregularity of $H$ on $K \backslash\{i d\}$ places strong restrictions on both $H$ and $K$, as the following lemma and corollary demonstrate. We include a proof based on [27] since it is so short and indicates the importance of the underlying set $X$ being finite.

Lemma 2.4 Let $A$ be any finite group. Suppose that $\varphi \in \operatorname{Aut}(A)$ is an involution that is fixed-point free on $A \backslash\{i d\}$. Then $\varphi=\alpha$ and so $A$ is Abelian. Necessarily $|A|$ is odd.

Proof Consider the mapping $x \mapsto x^{-1} \varphi(x)$ for $x \in A$. Suppose that $x^{-1} \varphi(x)=$ $y^{-1} \varphi(y)$ holds for some $x, y \in A$. Then $y x^{-1}=\varphi(y) \varphi(x)^{-1}=\varphi\left(y x^{-1}\right)$. Since $\varphi$ is fixed-point free on $A \backslash\{i d\}$, we have $y=x$. Thus, the mapping is injective, and since 
$A$ is finite, it is also bijective. Hence, every element $z \in A$ can be written as $x^{-1} \varphi(x)$ for some $x \in A$, yielding

$$
\varphi(z)=\varphi\left(x^{-1}\right) \varphi^{2}(x)=\varphi(x)^{-1} x=z^{-1}
$$

Thus, $\alpha=\varphi \in A$, and so $A$ is Abelian. Since $\varphi$ is fixed-point free, all nonidentity elements of $A$ can be paired with their (distinct) inverses, and so $|A|$ must be odd.

We apply this lemma to the kernel of a Frobenius (permutation) group.

Corollary 2.5 If $G=H K$ is a Frobenius group and $|H|$ is even, then $K$ is Abelian and $|K|$ is odd. Moreover, $\alpha$ is the unique involution in $H$.

Recall that a finite group is nilpotent when it is the direct product of its Sylow subgroups. Two more important properties of Frobenius groups are the following:

Theorem 2.6 (Thompson [31]) The kernel of a Frobenius group is nilpotent.

Remark 2.7 Theorems 2.2 and 2.6, as well as Lemma 2.4, fail for infinite groups (see [5]).

Theorem 2.8 (Burnside) The Sylow p-subgroup of the complement of a Frobenius group is cyclic when $p$ is odd, and is cyclic or generalized quaternion when $p=2$ (cf. [27] Theorem 18.1(iv)).

The following lemma shows that both a characteristic subgroup $N$ of a kernel $K$ and its quotient $K / N$ are also kernels of (different) Frobenius groups.

Lemma 2.9 Suppose that $G=H K$ is a Frobenius group and $N$ is a characteristic subgroup of $K$. Then both $H N$ and $H(K / N)$ are Frobenius groups. In particular, $|H|$ divides both $|N|-1$ and $|K / N|-1$.

Proof Since conjugation by $H$ leaves $N$ invariant, $H$ acts semiregularly on $N \backslash\{i d\}$. Hence, $H N$ is a Frobenius group, and so $|H|$ divides $|N|-1$. For $K / N$, the only $N$-coset fixed by conjugation by $H$ is the coset $N$ itself, since otherwise $|H|$ would divide the size of such a coset, namely $|N|$, which is coprime to $|N|-1$. Thus, the induced action by $H$ on $(K / N) \backslash\{N\}$ is semiregular, so $H(K / N)$ is a Frobenius group and $|H|$ divides $|K / N|-1$.

Corollary 2.10 Let $G=H K$ be a Frobenius group. Suppose that $\left|\operatorname{Syl}_{p}(K)\right|=p^{n}$ for some $n>1$. Then either $\operatorname{Syl}_{p}(K)$ is an elementary Abelian $p$-group or $|H|$ divides $p^{m}-1$ for some $m \leq n / 2$. If $n=3$, then $H$ is cyclic.

Proof Let $M=\operatorname{Syl}_{p}(K)$, so $|M|=p^{n}$. Since $K$ is nilpotent, $M$ is characteristic in $K$, and so $H M$ is a Frobenius group by Lemma 2.9. If $M$ is not an elementary Abelian $p$-group, then it contains a characteristic subgroup $N$. Since either $|N|$ or $p^{n} /|N|$ equals $p^{m}$ for some $m \leq n / 2$, a second application of Lemma 2.9 yields that $|H|$ divides $p^{m}-1$. When $n=3$, either $N$ or $M / N$ is the cyclic group $C_{p}$. Since $H$ injects into $\operatorname{Aut}\left(C_{p}\right)$ which is cyclic, $H$ is also cyclic. 
Corollary 2.11 Let $G=H K$ be a Frobenius group. For each prime divisor $p$ of $|K|$, there is an elementary Abelian p-group $N \leq K$ such that $H N$ is a Frobenius group.

Proof Since $K$ is nilpotent by Theorem 2.6, for any prime divisor $p$ of $|K|$, the Sylow $p$ - subgroup $\operatorname{Syl}_{p}(K)$ is characteristic. Every $p$-group has a nontrivial center, whose elements of order $p$ form a characteristic elementary Abelian $p$-group $N \leq \operatorname{Syl}_{p}(K)$. Now apply Lemma 2.9.

Example 2.12 By Corollary 2.11, every Frobenius group has within it a Frobenius subgroup that fits the following description: For some prime $p$, let $K$ be an elementary Abelian p-group. For some $n \geq 1, K$ may be regarded as a vector space of dimension $n$ over the finite field $\operatorname{GF}(p)$. Let $H$ be any subgroup of the general linear group $\operatorname{GL}(n, p)$ such that no nonidentity matrix $A$ in $H$ has an eigenvalue equal to 1 (i.e., $A x=x$ if and only if $x=\mathbf{0})$. Then $H K$ is a Frobenius group.

The case where $H$ is cyclic plays a prominent role in Sects. 4 and 5. Although one might think of a generator $h \in H$ as a "rotation" of $K$, it is also possible that the action of $h$ is multiplication by a scalar. Note that if we think of $K$ as the additive group of the field $\operatorname{GF}\left(p^{n}\right)$, then multiplication by a generator of the multiplicative group $\operatorname{GF}\left(p^{n}\right)^{*}$ gives a Frobenius group $G=H K$ where $|H|=|K|-1$.

Notation Because of the importance of the vector space model of Example 2.12, whenever $K$ is Abelian, we will use + to denote the group operation of $K$ and $\mathbf{0}$ to denote its identity element. In this context, a cyclic subgroup of $K$ of order $n$ will be denoted by $\mathbb{Z}_{n}$ rather than by $C_{n}$. However, for $H$, where the operation is composition of functions, we retain the notation $C_{n}$ for the cyclic group of order $n$.

\section{Products}

A significant difference between the solutions of the GRR problem and the GFR problem lies in the role of graph products. In brief, GRRs are product friendly; GFRs are not. The standard and best-known graph products are Cartesian, strong, categorical (also called "weak" or "Kronecker"), and lexicographic. For their definitions and basic properties, the reader is referred to [17] or [18]. All four of these graph products are associative, and the first three products are commutative (i.e., $\Gamma_{1} \circ \Gamma_{2} \cong \Gamma_{2} \circ \Gamma_{1}$ ). Another graph product, one that arises in Sect. 4.2, is defined as follows:

Definition 3.1 The deleted lexicographic product $\Gamma_{1} \odot \Gamma_{2}$ of graphs $\Gamma_{1}$ and $\Gamma_{2}$ has vertex set $V \Gamma_{1} \times V \Gamma_{2}$ and edges $\left\{\left(x_{1}, x_{2}\right),\left(y_{1}, y_{2}\right)\right\}$ if and only if

$$
\begin{array}{r}
x_{1}=y_{1} \text { and }\left\{x_{2}, y_{2}\right\} \in E \Gamma_{2}, \text { or } \\
\left\{x_{1}, y_{1}\right\} \in E \Gamma_{1} \text { and } x_{2} \neq y_{2} .
\end{array}
$$

This product is not commutative. It is subassociative, in the sense that $E\left(\left(\Gamma_{1} \odot \Gamma_{2}\right) \odot\right.$ $\left.\Gamma_{3}\right) \subset E\left(\Gamma_{1} \odot\left(\Gamma_{2} \odot \Gamma_{3}\right)\right)$ if the edge sets of the factors are not empty.

It was shown in [32] that if groups $G_{1}$ and $G_{2}$ other than $C_{2}$, admit GRRs $\Gamma_{1}$ and $\Gamma_{2}$, respectively, then $\Gamma_{1} \odot \Gamma_{2}$ is a GRR for the group $G_{1} \times G_{2}$. The deleted 
lexicographic product may be replaced by the any of the three commutative products when the factors $\Gamma_{1}$ and $\Gamma_{2}$ are relatively prime with respect to that product, that is, there exists no nontrivial graph $\Theta$ such that (for $i=1,2) \Gamma_{i}=\Delta_{i} \circ \Theta$ for some nontrivial graph $\Delta_{i}$ (cf. [18], Corollary 4.21).

By contrast, for Frobenius groups we have:

Lemma 3.2 Let $\left(G_{1}, X\right)$ and $\left(G_{2}, Y\right)$ be vertex-transitive permutation groups, where $X$ and $Y$ are finite sets, each having at least two elements. Then $\left(G_{1} \times G_{2}, X \times Y\right)$ in the product action either is regular or contains a nonidentity permutation that fixes two distinct elements of $X \times Y$. In either case, $\left(G_{1} \times G_{2}, X \times Y\right)$ is not Frobenius.

Proof If both $\left(G_{1}, X\right)$ and $\left(G_{2}, Y\right)$ are regular, then clearly so is $\left(G_{1} \times G_{2}, X \times Y\right)$. Without loss of generality, suppose that $\left(G_{2}, Y\right)$ is not regular, and so there exists $\varphi \in G_{2} \backslash\left\{i d_{G_{2}}\right\}$ that fixes some $y \in Y$. But then the nonidentity permutation $\left(i d_{G_{1}}, \varphi\right)$ fixes the point $(x, y)$ for every $x \in X$. Hence, $\left(G_{1} \times G_{2}, X \times Y\right)$ cannot be a Frobenius group.

Theorem 3.3 Let $\Gamma_{1}$ and $\Gamma_{2}$ be nontrivial graphs and let $\circ$ be any of the five aforementioned products. Then $\Gamma_{1} \circ \Gamma_{2}$ is not a GFR of any Frobenius group.

Proof If o represents the lexicographic or the deleted lexicographic product, then given any nontrivial $\varphi \in \operatorname{Aut}\left(\Gamma_{2}\right)$ and any vertex $x_{0} \in V \Gamma_{1}$, the function mapping $\left(x_{0}, y\right)$ to $\left(x_{0}, \varphi(y)\right)$ and fixing $(x, y)$ for all $x \neq x_{0}$ is a nontrivial automorphism that fixes more than one vertex. Thus, we do not have a GFR.

For the other products, if $\Gamma_{1}$ and $\Gamma_{2}$ are relatively prime with respect to $\circ$, then $\operatorname{Aut}\left(\Gamma_{1} \circ \Gamma_{2}\right)$ is the product action of $\operatorname{Aut}\left(\Gamma_{1}\right) \times \operatorname{Aut}\left(\Gamma_{2}\right)$. By Lemma 3.2, we do not have a GFR. If these factors are not relatively prime, then suppose that $\Gamma_{i}=\Delta_{i} \circ \Theta$ for $i=1,2$. The function that maps $(x, y)$ to $(y, x)$ if both $x, y \in V \Delta$ and fixes all other vertices of $\Gamma_{1} \circ \Gamma_{2}$ is a nontrivial automorphism that fixes at least two vertices. Again $\Gamma_{1} \circ \Gamma_{2}$ is not a GFR.

Remark 3.4 Suppose we are given Frobenius groups $H K_{1}$ and $H K_{2}$. Although their product $H K_{1} \times H K_{2}$ is not a Frobenius group, there nonetheless exists a Frobenius group $H\left(K_{1} \times K_{2}\right)$ where the action of $H$ is defined by $h\left(x_{1}, x_{2}\right)=\left(h\left(x_{1}\right), h\left(x_{2}\right)\right)$.

\section{Construction of GFRs}

When a graph $\Gamma$ is a GFR of a Frobenius group $G=H K$, then, as in the case of GRRs, the graph $\Gamma$ must be a Cayley graph $\operatorname{Cay}(K, S)$ where $S$ is an inverse closed subset of $K \backslash\{i d\}$ that generates $K$. This is because $K$ acts regularly on the vertex set of $\Gamma$ and, by the classical result by Sabidussi [28], a graph $\Gamma$ is a Cayley graph for a group $K$ if and only if $\operatorname{Aut}(\Gamma)$ contains a regular subgroup isomorphic to $K$. Hence, if we wish to construct a GFR of $G$, we must construct a Cayley graph Cay $(K, S)$ whose automorphism group, as a permutation group of its vertex set, is the permutation group $G$. Toward that end, the following basic result is essential.

Proposition 4.1 [32, Lemma 3] Given any Cayley graph Cay $(K, S)$, the set of group automorphisms of $K$ that fix $S$ setwise is a subgroup of the stabilizer in $\operatorname{Aut}(\operatorname{Cay}(K, S))$ of the identity vertex $i d$. 


\subsection{Unwanted automorphisms}

Given a Frobenius group $G=H K$ and any Cayley graph Cay $(K, S)$, it holds that $G \leq \operatorname{Aut}(\operatorname{Cay}(K, S))$ if and only if $S$ is invariant under conjugation by $H$ (since the elements of $S$ are the vertices adjacent to the identity). Thus, a necessary condition for a graph to be a GFR of $G$ is that it be a Cayley graph for $K$ whose connection set $S$ is invariant under conjugation by $H$; that is, $S$ is closed under inverses and is a union of orbits of the action of $H$ on $K$. Our challenge is to find a connection set $S$ for which Cay $(K, S)$ admits no unwanted automorphisms in the stabilizer of the vertex $i d$. An unwanted automorphism must be either:

- a group automorphism of $K$ not in $H$ that leaves $S$ invariant, or

- a graph automorphism of $\operatorname{Cay}(K, S)$ that fixes the vertex labeled id but which is not a group automorphism of $K$.

The following lemma offers an example of unwanted group automorphisms.

Lemma 4.2 Suppose $G=H K$ is a Frobenius group where $|H|$ is odd and $K$ is Abelian but not an elementary Abelian 2-group. Then $G$ admits no GFR.

Proof Suppose that Cay $(K, S)$ is a GFR of $G$ where $K$ is Abelian but not an elementary 2-group. Then the inversion function $\alpha$ is a nontrivial group automorphism of $K$ that fixes $S$ setwise. By Proposition 4.1, $\alpha$ is a graph automorphism of $\operatorname{Cay}(K, S)$ that fixes the vertex $i d$. But $\alpha \notin H$ because $|H|$ is odd.

On the other hand, when $|H|=2$, and so (by Corollary 2.5) $K$ is Abelian of odd order and $H=\langle\alpha\rangle$, the work of finding GFRs has already been done by Imrich and Watkins [20] in the context of "approximating" GRRs:

Theorem 4.3 [20] For any Abelian group $K$ of odd order other than $\left(\mathbb{Z}_{3}\right)^{2}$ and $\left(\mathbb{Z}_{3}\right)^{3}$, there is a Cayley graph Cay $(K, S)$ whose only nontrivial automorphism fixing id is inversion. (Thus, if $G=H K$ is a Frobenius group with $|H|=2$, then $G$ admits a GFR unless $K$ is $\left(\mathbb{Z}_{3}\right)^{2}$ or $\left(\mathbb{Z}_{3}\right)^{3}$.)

The following lemma exemplifies the problem of unwanted automorphisms of the second kind, namely graph automorphisms that are not group automorphisms:

Lemma 4.4 Suppose that $G=H K$ where $|K| \geq 4$ and $|H|=|K|-1$. Then $G$ admits no GFR. In this case, $K$ is an elementary Abelian p-group.

Proof Suppose that $\Gamma=\operatorname{Cay}(K, S)$ is a GFR of $G$. If $|H|=|K|-1$ holds, then since $H$ acts semiregularly on $K \backslash\{i d\}$, it follows that $H$ also acts transitively on $K \backslash\{i d\}$, and so $S=K \backslash\{i d\}$ itself is the only $H$-orbit. But then $\operatorname{Cay}(K, S)$ is the complete graph on $|K|$ vertices, which clearly has unwanted graph automorphisms when $|K| \geq 4$.

Since $|H|=|K|-1$ must divide the order of any characteristic subgroup of $K$, it follows that no such proper characteristic subgroup exists. Since $K$ is nilpotent by Theorem 2.6, $K$ must be a $p$-group, and the only $p$-groups having no characteristic proper subgroup are elementary Abelian $p$-groups. (Note that this occurs in particular when $H$ is the cyclic multiplicative group of the field $G F\left(p^{n}\right)$.) 
It is an elementary exercise to prove that at least one of a graph $\Gamma$ and its complement $\bar{\Gamma}$ is connected. For GFRs, which are connected by definition, one can say more.

Lemma 4.5 Let $\Gamma$ be a GFR with $|V \Gamma| \geq 4$. Then its complement $\bar{\Gamma}$ is connected.

Proof Suppose that $\Gamma$ is a GFR but that $\bar{\Gamma}$ is not connected. Since $\bar{\Gamma}$ is vertex-transitive, it can be written as a lexicographic product $\bar{\Gamma} \cong \overline{K_{n}} \circ \Delta$, where $n \geq 2$ and $\Delta$ is connected and vertex-transitive. As $\Gamma$ is not complete (by Lemma 4.4), we have $|V \Delta| \geq 2$, and so by Theorem 3.3, Aut $(\bar{\Gamma})=\operatorname{Aut}(\Gamma)$ is not a Frobenius group.

Remark 4.6 Suppose that $\Gamma=\operatorname{Cay}(K, S)$ is a GFR of a group $G=H K$. Lemma 4.5 tells us that the set $\bar{S}=K \backslash(S \cup\{i d\})$ also generates $K$. Hence, $\bar{\Gamma}=\operatorname{Cay}(K, \bar{S})$ is also a GFR of $G$. We may therefore always assume that $|S| \leq(|K|-1) / 2$.

\subsection{Normal Cayley graphs and cyclic kernels}

We seek criteria for a graph automorphism of $\operatorname{Cay}(K, S)$ that fixes $i d$ to be a group automorphism of $K$.

Definition 4.7 (Cf. [38]) Given a Cayley $\operatorname{graph} \operatorname{Cay}(K, S)$, let $K_{L}=\left\{\lambda_{k}: k \in K\right\}$, where $\lambda_{k}$ is left translation by $k$, that is, $\lambda_{k}(x)=k x$ for all $x \in K$. By Cayley's theorem, $K_{L}$ is a regular subgroup of $\operatorname{Aut}(\operatorname{Cay}(K, S))$. The Cayley graph Cay $(K, S)$ is normal if $K_{L}$ is a normal subgroup of $\operatorname{Aut}(\operatorname{Cay}(K, S))$.

Remarks 1. It is possible to have Cay $(K, S)=\mathrm{Cay}\left(K^{\prime}, S^{\prime}\right)$ for nonisomorphic groups $K$ and $K^{\prime}$ while the graph is normal with respect to only one of the groups. Our convention is that when we write "Cay $(K, S)$ is normal," we understand the normality to be with respect to the given group $K$.

2. If Cay $(K, S)$ is a GFR for $G=H K$, then $\operatorname{Cay}(K, S)$ must be normal since $K$ is normal in $G=\operatorname{Aut}(\operatorname{Cay}(K, S))$.

The following result characterizes normal Cayley graphs. It implies that if a candidate to be a GFR is normal, then it admits no unwanted graph automorphisms.

Lemma 4.8 Let $A=\operatorname{Aut}_{i d}(\operatorname{Cay}(K, S))$ and suppose $\varphi \in A$. Then $\varphi$ is a group automorphism of $K$ if and only if it normalizes $K_{L}$. In particular, all elements of $A$ are group automorphisms if and only if $\operatorname{Cay}(K, S)$ is normal.

Proof Suppose that the graph automorphism $\varphi$ normalizes $K_{L}$. Then for any $x \in K$, there exists $z \in K$ such that $\varphi \lambda_{x}=\lambda_{z} \varphi$, that is, $\varphi(x y)=z \varphi(y)$ for all $y \in K$. Setting $y=i d$ gives us $z=\varphi(x)$, and so $\varphi(x y)=\varphi(x) \varphi(y)$ for all $y$. Since $x$ was arbitrary, $\varphi$ is a group automorphism. In particular, if $\operatorname{Cay}(K, S)$ is normal, then all elements of $A$ are group automorphisms.

Conversely, suppose $\varphi \in A$ is also a group automorphism, that is, $\varphi(x y)=$ $\varphi(x) \varphi(y)$ holds for all $x, y \in K$. Thus, $\varphi\left(\lambda_{x}(y)\right)=\lambda_{\varphi(x)} \varphi(y)$, that is, $\varphi$ conjugates $\lambda_{x}$ to $\lambda_{\varphi(x)}$. Since $x$ is arbitrary, $\varphi$ normalizes $K_{L}$. In particular, since $\operatorname{Aut}(\operatorname{Cay}(K, S))=A K_{L}$, if all elements of $A$ are group automorphisms, then $\operatorname{Cay}(K, S)$ is normal. 
A Cayley graph of a cyclic group is called a circulant. The simplest example of a circulant is the graph consisting of an $n$-cycle. When $n$ is odd, the $n$-cycle is a GFR for the dihedral group $D_{n}$, with $K \cong \mathbb{Z}_{n}$ and $H=\langle\alpha\rangle$. On the other hand, when $n$ is even, the stabilizer of any vertex also fixes its antipodal vertex, and so the $n$-cycle is not a GFR for $D_{n}$; in fact, when $n$ is even, $D_{n}$ as a permutation group on $n$ points is simply not a Frobenius group.

Recall that Abelian groups are written additively, and so a circulant has the form Cay $\left(\mathbb{Z}_{n}, S\right)$, where $\mathbf{0} \notin S=-S$. We understand the "inverting function" to be $\alpha$ : $x \mapsto-x$.

Theorem 4.9 Let $G=H \mathbb{Z}_{n}$ be a Frobenius group, where $|H|<n-1$ and $|H|$ is even. Let $S$ be the orbit under $H$ of any generator of the additive group $\mathbb{Z}_{n}$. Then $\operatorname{Cay}\left(\mathbb{Z}_{n}, S\right)$ is a GFR for $G$.

Proof By Corollary 2.5, $n$ is odd and $\alpha \in H$. Hence, $S$ is closed with respect to negation. Since $S$ generates $G$, it is a connection set for the graph $\operatorname{Cay}\left(\mathbb{Z}_{n}, S\right)$. By hypothesis, $H$ acts transitively on the neighbors of the vertex labeled $\mathbf{0}$, and so $\operatorname{Cay}\left(\mathbb{Z}_{n}, S\right)$ is an arc-transitive circulant. The hypothesis of a theorem of Cai Heng Li ([22], Theorem 1.3, and see also [21]) is now satisfied, from which it follows that either

- $\operatorname{Cay}\left(\mathbb{Z}_{n}, S\right)$ is a normal circulant, or

- there exists an arc-transitive circulant $Y$ of order $m$ such that $n=m b$ with $b, m \geq 2$, and $\operatorname{Cay}\left(\mathbb{Z}_{n}, S\right)$ is either the lexicographic product or the deleted lexicographic product of $Y$ with $\overline{K_{b}}$.

If Cay $\left(\mathbb{Z}_{n}, S\right)$ were to have the form of the second option, then by Theorem 3.3, Cay $\left(\mathbb{Z}_{n}, S\right)$ could not be a GFR. We now eliminate that option. Since $n$ is odd, we have $m, b \geq 3$. If $Y$ has valence $r$, then the valence $|H|$ of Cay $\left(\mathbb{Z}_{n}, S\right)$ is either $r b$ or $r(b-1)$, depending upon which product applies. In either case, we have $|H| \geq 2(b-1)$, since $r \geq 2$. Since all subgroups of $\mathbb{Z}_{n}$ are characteristic and $b$ divides $n$, we have that $|H|$ divides $b-1$ by Lemma 2.9, giving the contradiction $2(b-1) \leq|H| \leq b-1$.

Hence, Cay $\left(\mathbb{Z}_{n}, S\right)$ is normal and so admits no unwanted graph automorphisms (by Lemma 4.8). Since group automorphisms of $\mathbb{Z}_{n}$ consist of multiplication by the units of $\mathbb{Z}_{n}$, we may assume by hypothesis that $S$ is the orbit of 1 in $\mathbb{Z}_{n}$. Multiplication by any unit $u \in \mathbb{Z}_{n}$ leaves $S$ invariant if and only if $u=u \cdot 1 \in S$. Hence, there are no unwanted group automorphisms, making $\operatorname{Cay}\left(\mathbb{Z}_{n}, S\right)$ a GFR for $G$.

Remark When $n$ is not prime, the automorphism group of the cyclic group $\mathbb{Z}_{n}$ need not be cyclic. However, in Theorem 4.9 the complement $H$ must be cyclic, since $H$ leaves invariant each $p$-Sylow subgroup of $\mathbb{Z}_{n}$ and the automorphism group of any cyclic group of prime power order is cyclic.

\subsection{Dihedral stabilizers}

In addition to normality, there is a second, less well-known criterion for recognizing group automorphisms in $\operatorname{Aut}_{i d}(\mathrm{Cay}(K, S))$. First observe that for any automorphism 
$\varphi$ of $\operatorname{Cay}(K, S)$ and any $y$ in $K$, there is a local permutation of $S$, denoted by $\varphi_{y}$, since $\varphi$ maps the arcs emanating from $y$ bijectively to the arcs emanating from $\varphi(y)$, and these arcs bear a labeling by the elements of $S$. Specifically, if $x \in S$, then $\varphi_{y}(x)$ satisfies $\varphi(y) \varphi_{y}(x)=\varphi(y x)$. By definition, the local permutations of a left translation are the identity permutation on $S$.

Lemma 4.10 A graph automorphism $\varphi \in \operatorname{Aut}_{i d}(\operatorname{Cay}(K, S))$ is a group automorphism of $K$ if and only if $\varphi_{y}=\varphi_{i d}$ for all $y \in K$.

Proof Suppose $\varphi$ is a group automorphism. Then for all $x \in S$ and all $y \in K$, we have $\varphi(y x)=\varphi(y) \varphi(x)$. But for $x \in S$, we have $\varphi_{i d}(x)=\varphi(x)$. Thus, $\varphi_{y}=\varphi_{i d}$, since the local permutations of $\lambda_{\phi(y)}$ are the identity permutation on $S$. Conversely, suppose that $\varphi_{y}=\varphi_{i d}$ for all $y \in K$. For any $x \in S$, we have

$$
\varphi(y x)=\varphi(y) \varphi_{y}(x)=\varphi(y) \varphi_{i d}(x)=\varphi(y) \varphi(x) .
$$

For any $z \in K$, by induction on the distance in $\operatorname{Cay}(K, S)$ from $i d$ to $z$, one can replace $x$ by $z$ to obtain $\varphi(y z)=\varphi(y) \varphi(z)$.

Theorem 4.11 Let $G=H K$ be a Frobenius group with $H=C_{2 m}$ for $m>1$, and let $x \in K$. Suppose that the orbit $S=H x$ generates $K$ and that the stabilizer $\operatorname{Aut}_{\mathbf{0}}(\mathrm{Cay}(K, S))$ acts on $S$ as $C_{2 m}$ or $D_{2 m}$. Then $\mathrm{Cay}(K, S)$ is normal. Moreover, if $K$ contains a characteristic subgroup $N$ such that $N$ or $K / N$ is cyclic, then $\operatorname{Cay}(K, S)$ is a GFR for $G$.

Proof Recall (Corollary 2.5) that $K$ is Abelian and $|K|$ is odd. Since $S=H x$, the action of $H$ restricted to $S$ is regular and, in fact, defines a cycle of length $|H|$ generated by some $h \in H$. This action defines a cyclic order on $S$, which we regard as a rotation of the arcs emanating from any vertex. By hypothesis, for any $y \in K$ and any graph automorphism $\varphi$, the local permutation $\varphi_{y}$ either preserves or reverses the rotation at $y$.

We first prove that the action of $A=\operatorname{Aut}_{0}(\operatorname{Cay}(K, S))$ on $S$ is faithful, namely that if $\varphi \in A$ fixes $S$ pointwise, then $\varphi=i d$ holds. Choose any $y \in S$ and let $z=h(y)$. Consider the 4-cycle ("parallelogram") in Cay $(K, S)$ whose vertices in cyclic order are $\mathbf{0}, y, z, y+z=z+y$. Suppose that $\varphi$ reverses the rotation at both vertex $y$ and vertex $z$, that is, $\varphi$ maps the $\operatorname{arc}(y, y+z)$ to the $\operatorname{arc}\left(y, y+h^{-1}(z)\right)$ and maps the arc $(z, z+y)$ to the arc $\left(z, z+h^{-1}(y)\right)$. Thus, $\varphi(y+z)=y+h^{-1}(y)$ and $\varphi(z+y)=z+h(z)$, that is, $y+h^{-1}(y)=z+h(z)$. Setting $w=h^{-1}(y)$ yields $h(w)+w=z+h(z)$, and so $h(z-w)=w-z$, forcing $h^{2}(z-w)=z-w$ and hence $m=1$, a contradiction. Therefore, without loss of generality, $\varphi$ preserves the rotation at $y$. Since $\varphi$ fixes the arc labeled $y$, it fixes all arcs at vertex $y$, and so $\varphi$ fixes $y+z=z+y$. Hence, $\varphi$ also fixes the arcs labeled $y$ and $z$ at vertex $z$, and hence, $\phi$ fixes all arcs at $z$ as well. We conclude that $\varphi$ fixes all neighbors of $y$ and $z$. Since $y \in S$ was arbitrary, $\varphi$ fixes all vertices at distance 2 from $\mathbf{0}$. Replacing $\mathbf{0}$ by $y \in S$, we get that $\varphi$ fixes all vertices at distance 2 from $y$ and hence at distance 3 from $\mathbf{0}$. Continuing by induction on the distance from $\mathbf{0}$ in $\operatorname{Cay}(K, S)$, we conclude that $\varphi$ fixes every vertex, and so $A$ acts faithfully. 
The faithfulness of the action of $A$ on $S$ tells us that if $A=C_{2 m}$, then Cay $(K, S)$ is a GFR for $G=H K$. What remains to show is that if $A=D_{2 m}$, then $\operatorname{Cay}(K, S)$ is normal and that, if there is a characteristic subgroup $N \leq K$ such that $N$ or $K / N$ is cyclic, then $A=D_{2 m}$ is impossible.

Suppose $\varphi \in A$ preserves the rotation at $\mathbf{0}$. We claim that, for all $y \in K, \varphi_{y}$ preserves the rotation at $y$. Whether the action of $A$ is $C_{2 m}$ or $D_{2 m}$, it holds that the restriction $\varphi\left|S=h^{i}\right| S$ for some $i$, and hence, $\varphi=h^{i}$ everywhere. In particular, $\varphi$ preserves the rotation at all vertices. Using the action of $K_{L}$, which preserves the rotation, it follows that, if $\varphi$ preserves the rotation at any vertex $y$, then it preserves it everywhere. Thus, if $\varphi$ reverses the rotation at any one vertex, it reverses the rotation everywhere.

Once it is known whether the nonidentity automorphism $\varphi$ preserves the rotation everywhere or reverses the rotation everywhere, the local permutation $\varphi_{y}$ is determined by its action at any single element of $S$. Thus, $\varphi_{x}=\varphi_{0}$ for any $x \in S$. Inductively as before, we get $\varphi_{y}=\varphi_{\mathbf{0}}$ for all $y \in K$. Hence, $\varphi$ is a group automorphism by $\operatorname{Lemma} 4.10$, and $\operatorname{Cay}(K, S)$ is normal by Lemma 4.8 .

What remains to show is that if $K$ contains a characteristic subgroup $N$ such that $N$ or $K / N$ is cyclic, then $A=C_{2 m}$. Suppose instead that $A=D_{2 m}$. Since $H=C_{2 m}$ injects into both $\operatorname{Aut}(N)$ and $\operatorname{Aut}(K / N)$, so must $A=D_{2 m}$, because $A$ contains $H$ and there exists no homomorphism from $D_{2 m}$ onto $C_{2 m}$ for $m>1$. But $D_{2 m}$ cannot inject into $\operatorname{Aut}(N)$ and $\operatorname{Aut}(K / N)$, since at least one of them is Abelian. Thus, $A=C_{2 m}$, so $\operatorname{Cay}(K, S)$ is a GFR for $G$.

\subsection{GFRs when $H=C_{4}$ or $C_{6}$}

Throughout this subsection, we are assuming that $G=H K$ is a Frobenius group and $|H|$ is even. Hence, $K$ is Abelian, $|K|$ is odd, and $H$ contains a unique involution, namely $\alpha$. Thus, if $|H|=4$ or 6 , then $H$ is cyclic, and we fix the notation that $H$ is generated by an element $h$ of order 4 or 6 accordingly.

Theorem 4.12 Suppose that $|H|=4$ and $|K|>9$. Let $x \in K$ and suppose that the orbit $S=H x$ generates $K$. Then $\mathrm{Cay}(K, S)$ is normal with vertex stabilizer $D_{4}$ or $C_{4}$. In particular, if $K$ is cyclic or has the form $\mathbb{Z}_{n} \times \mathbb{Z}_{m n}$ for some $m \geq 3$, then $\operatorname{Cay}(K, S)$ is a GFR for $G$.

Proof Since $H=C_{4}$, the orbit $S=H x$ has the form $\{x, y,-x,-y\}$, where $y=h(x)$. Figure 1 shows the 2-ball of $\operatorname{Cay}(K, S)$ centered at $\mathbf{0}$. Since $|K|>9$, both $x$ and $y$ have order at least 5 , and hence, all the vertices shown in the figure are distinct. Clearly $\operatorname{Aut}(\operatorname{Cay}(K, S))$ restricted to the subgraph induced by this 2-ball is the dihedral group $D_{4}$. Therefore, by Theorem 4.11 , Cay $(K, S)$ is normal with vertex stabilizer $C_{4}$ or $D_{4}$.

If $K$ is cyclic, a GFR of $G$ exists by Theorem 4.9. If $K$ has the form $\mathbb{Z}_{n} \times \mathbb{Z}_{m n}$ for some $m \geq 3$, then the elements of $K$ of order $n$ form a characteristic subgroup $N$ with cyclic quotient $K / N=\mathbb{Z}_{m}$, and $\operatorname{Cay}(K, S)$ is a GFR of $G$ by Theorem 4.11.

When $K=\left(\mathbb{Z}_{n}\right)^{2}$, a single generating orbit is insufficient to destroy the "reflections" in $D_{4}$, and so an additional orbit must be included in the connection set to produce a GFR. 


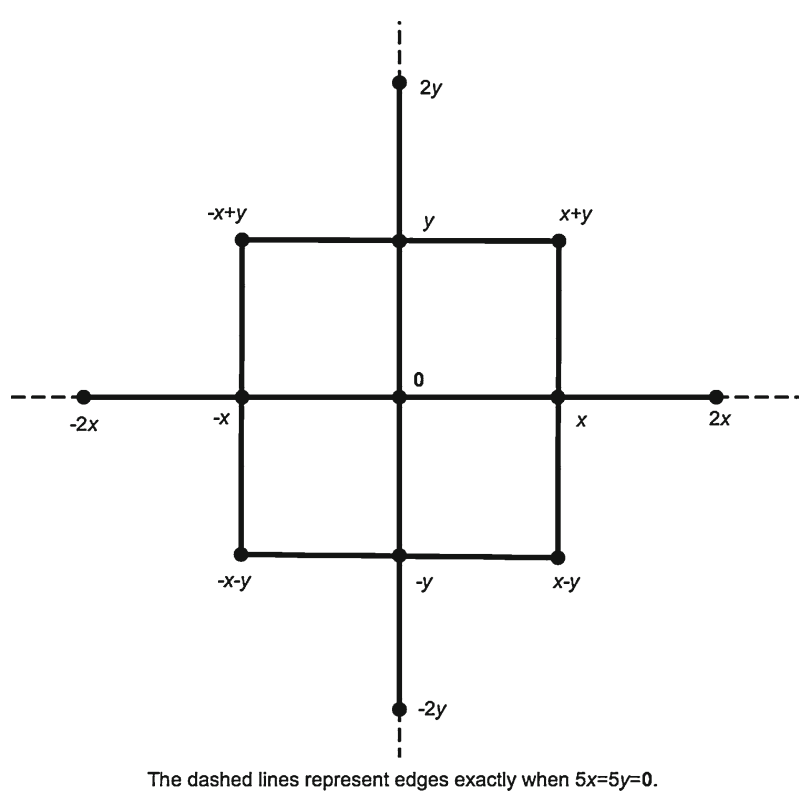

Fig. 1 The 2-ball centered at $\mathbf{0}$ of $\operatorname{Cay}(K, S)$ in Theorem 4.12

Theorem 4.13 Suppose that $|H|=4$ and $K=\left(\mathbb{Z}_{n}\right)^{2}$ for $n>3$. Let $x \in K$, let $H=\langle h\rangle$ and suppose that the orbit $H x$ generates $K$. Then $\operatorname{Cay}(K, S)$ where $S=H x \cup H(2 x+h(x))$ is a GFR for $G=H K$.

Proof Observe that the order $n$ of both $x$ and $y=h(x)$ is an odd integer at least 5 . For $i=1,2$, let $R_{i}$ denote the set of vertices in the $i$-sphere about $\mathbf{0}$ of $\operatorname{Cay}(K, S)$. Let $T$ denote the subset of vertices in $R_{2}$ that are adjacent to at least two vertices of $R_{1}$, and let $\Theta$ denote the subgraph of $\operatorname{Cay}(K, S)$ induced by $\{\boldsymbol{0}\} \cup R_{1} \cup T$. Clearly every automorphism of Cay $(K, S)$ fixes (setwise) each of $\{\mathbf{0}\}, R_{1}$, and $T$.

First suppose that $n>5$. Of the vertices in $T$, only those in the orbit $H(x+y)$ have four neighbors in $R_{1}$; all other vertices in $T$ have two or three neighbors in $R_{1}$, as shown in Fig. 2. (For example, $x+y$ is adjacent to $x,-x, y \in H x$ and to $2 x+y \in H(2 x+y)$.) Hence, the orbit $H(x+y)$ is invariant. Since each vertex in $H(x+y)$ has exactly three neighbors in $H x$ and one neighbor in $H(2 x+y)$, each of the two orbits contained in $S$ is invariant.

When $n=5$, we have the additional relation $(2 x+y)+(2 x+y)=-x+2 y \in$ $H(2 x+y)$. Concentrating only on $R_{1}$, we see that the orbit $H(2 x+y)$ induces a 4-cycle in $R_{1}$ (indicated by dashed lines in Fig. 2). On the other hand, $x$ cannot be expressed as a sum of two elements of $S$, and so the vertices of $H x$ have valence 0 in $R_{1}$. Again any automorphism fixing $\mathbf{0}$ leaves invariant each of $H(x)$ and $H(x+2 y)$.

By Lemma 4.8 and Theorem 4.11, Cay $(K,\{x, y,-x,-y\})$ is normal with stabilizer of 0 being either $C_{4}$ or $D_{4}$. In the latter case, there is a group automorphism $\sigma$ such that $\sigma(x)=x$ and $\sigma(y)=-y$. But then $\sigma(2 x+y)=2 x-y \notin S$. Therefore, $H$ is the (entire) stabilizer of $\mathbf{0}$, and so Cay $(K, S)$ a GFR for $G$. 


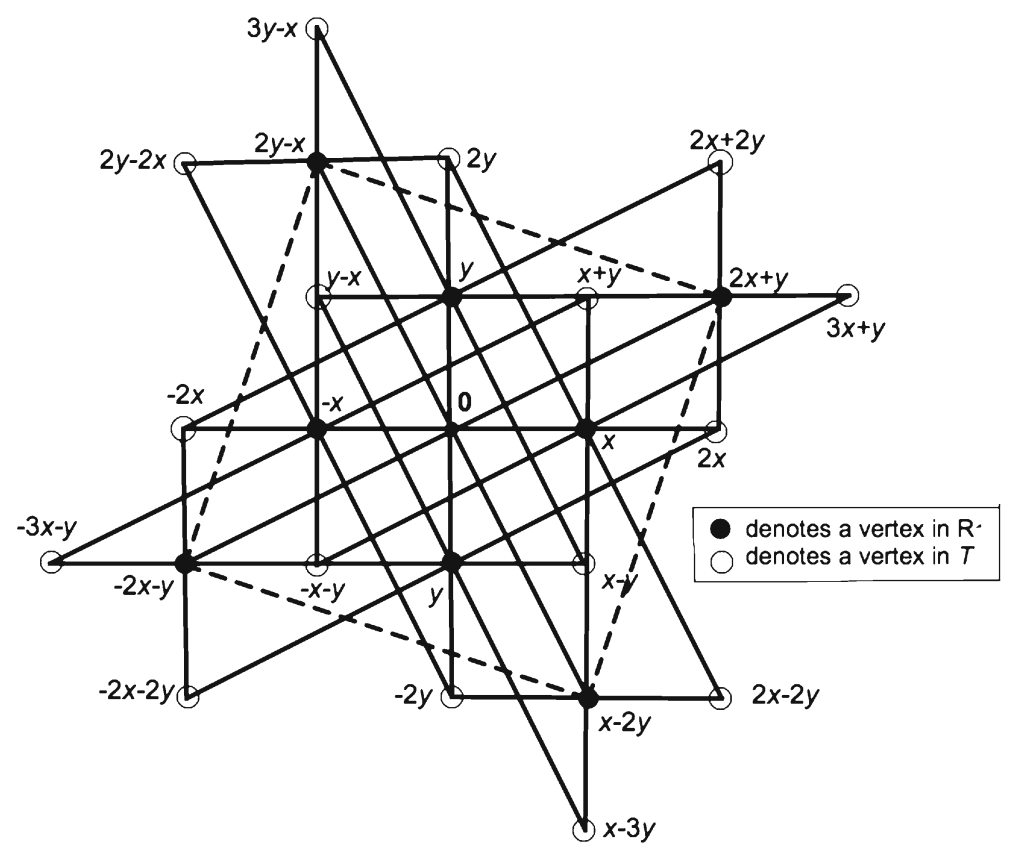

Dashed lines indicate additional edges in $R$. when $n=5$.

Fig. 2 The subgraph $\Theta$ in Theorem 4.13

Theorem 4.14 Suppose that $|H|=6$. Let $x \in K$ and suppose that the orbit $H x$ generates $K$. If $K \neq\left(\mathbb{Z}_{n}\right)^{2}$, then $\operatorname{Cay}(K, H x)$ is a GFR for $G=H K$. If $K=\left(\mathbb{Z}_{n}\right)^{2}$ where $n>5$, then Cay $(K, H x \cup H(2 x+h(x)))$ is a GFR for G.

Proof Observe that $h^{3}=\alpha$. In the ring of endomorphisms from $K$ to $K$, we have

$$
(h-\alpha)\left(h^{2}+\alpha h+\alpha^{2}\right)=h^{3}-\alpha=\mathbf{0} .
$$

If $(h-\alpha)(z)=\mathbf{0}$ for some $z \neq \mathbf{0}$, then $h(z)=-z$ and so $h^{2}(z)=-h(z)=z$, contrary to the definition of a Frobenius group. Thus, $h-\alpha$ is injective and hence is invertible. Applying $(h-\alpha)^{-1}$ to both sides of Eq. (4.1) yields $h^{2}+\alpha h+\alpha^{2}=\mathbf{0}$. Thus, $h^{2}(x)=h(x)-x$. In particular, $\{x, h(x)\}$ generates $K$, since $H x$ generates $K$. Thus, $K$ is 2-generated, and $K$ is either $\left(\mathbb{Z}_{n}\right)^{2}$ or $\mathbb{Z}_{n} \times \mathbb{Z}_{m n}$ with $m \geq 3$.

With $y=h(x)$, the orbit $H x$ becomes cyclically $(x, y,-x+y,-x,-y, x-y)$. The 1-sphere about $\mathbf{0}$ in $\operatorname{Cay}(K, H x)$ is a 6-cycle, namely the orbit under $H$ of the arc from $x$ to $y$ labeled by the generator $-x+y$. By Theorem 4.11, Cay $(K, H x)$ is normal with vertex stabilizer either $C_{6}$ or $D_{6}$. If $K \neq\left(\mathbb{Z}_{n}\right)^{2}$ for some $n$, then $K$ has a characteristic subgroup $N$ such that $N$ or $K / N$ is cyclic. Hence, Cay $(K, H x)$ is a GFR for $G$.

Now suppose $K=\left(\mathbb{Z}_{n}\right)^{2}$ with $n>5$ and let $S=H x \cup H(2 x+y)$. For ease of computation, observe that the action of $h$ on $\left(\mathbb{Z}_{n}\right)^{2}$ is given by the matrix $\left(\begin{array}{cc}0 & -1 \\ 1 & 1\end{array}\right)$. 


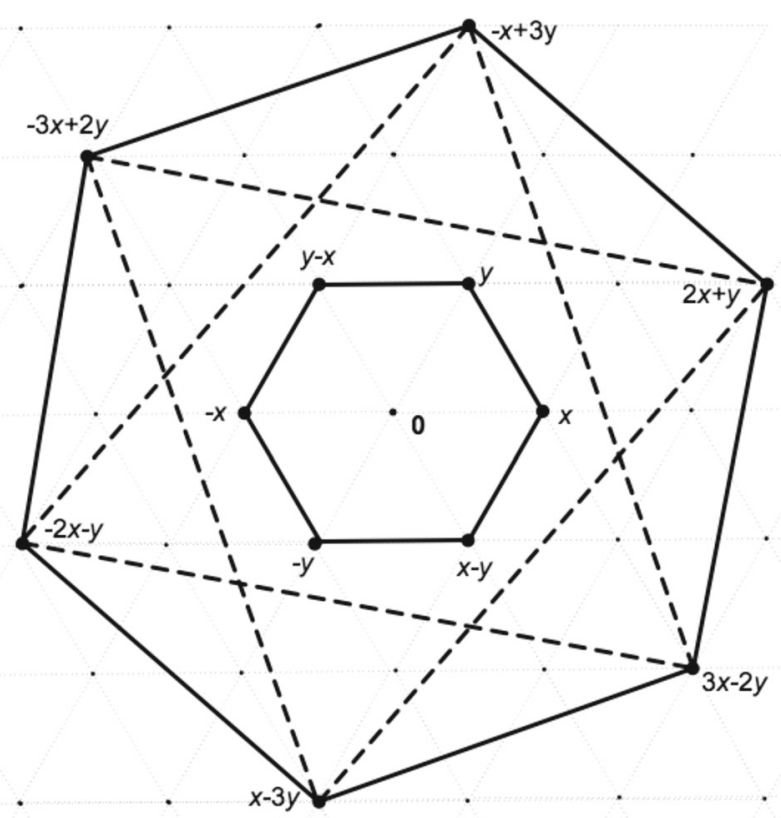

Dashed segments are edges exactly when $7 x=7 y=0$.

Fig. 3 The 1-sphere about $\mathbf{0}$ in $\operatorname{Cay}\left(\left(\mathbb{Z}_{n}\right)^{2}, S\right)$ in Theorem 4.14

One thus verifies that

$$
H(2 x+y)=\{ \pm(2 x+y), \pm(3 y-x), \pm(2 y-3 x)\}
$$

and so the 1-sphere about $\mathbf{0}$ now has the form of Fig. 3.

We next show the two $H$-orbits contained in $S$ are invariant under $H$. When $n=7$, this is immediate; in the subgraph induced by $S$, namely the 1-sphere about $\mathbf{0}$, one sees that the vertices in $H x$ have valence 2 while those in $H(2 x+y)$ have valence 4 . When $n>7$ all the vertices in this subgraph are 2-valent, and we argue as follows. Any vertex $z$ in the 2-sphere about $\mathbf{0}$ can be expressed as $z=s+t=t+s$ where $s, t \in S$. If $s \neq t$, then $z$ has at least two neighbors in $S$. Thus, the only way that $z$ can have exactly one neighbor in $S$ is when $z=2 s$ for some $s \in S$ and no other relation $z=s+t$ holds. Since $2 x=(2 x+y)+(-y)$, only vertices in the orbit $H(4 x+2 y)$ have this fortuitous property. Hence, the orbit $H(4 x+2 y)$ is invariant, which forces each orbit in $S$ to be invariant. The proof concludes exactly as does the proof of Theorem 4.13. Indeed, this same argument applies for $n>5$ in Theorem 4.13 .

\subsection{Subgroups of $\boldsymbol{H}$}

We have considered Frobenius groups associated with a characteristic subgroup of the kernel $K$. If $G=H K$ is a Frobenius group and $H^{\prime}$ is a nontrivial subgroup of $H$, 
then $H^{\prime} K$ is also a Frobenius group. It is natural to ask how GFRs for $H^{\prime} K$ might be related to GFRs for $H K$.

Proposition 4.15 Suppose that $G=H K$ is a Frobenius group and $H^{\prime}$ is a nontrivial subgroup of $H$. Suppose that $G^{\prime}=H^{\prime} K$ does not have a GFR because every candidate has either an unwanted graph automorphism that is not a group automorphism or an unwanted group automorphism that fixes some nonidentity element of $K$. Then $G$ does not have a GFR for the same reason as $G^{\prime}$.

Proof If $S$ is a union of orbits of $H$, then $S$ is also a union of orbits of $H^{\prime}$. By hypothesis, Cay $(K, S)$ is not a GFR for $G^{\prime}=H^{\prime} K$ because it has either an unwanted graph automorphism or an unwanted group automorphism that fixes a nonidentity element of $K$. Thus, $\operatorname{Cay}(K, S)$ is not a GFR for $G=H K$.

\section{GFRs of groups of order $\leq \mathbf{3 0 0}$}

In this section, we classify all Frobenius groups of order at most 300 with regard to whether they admit a GFR. Many of the ideas from the previous sections are encountered in the process. This exercise motivates various questions considered in Sect. 6.

We have already established two classes of Frobenius groups $G=H K$ that never admit a GFR:

- $|H|$ is odd and $K$ is Abelian but not an elementary Abelian 2-group (Lemma 4.2); and

- $K$ is an elementary Abelian $p$-group, $|K| \geq 4$, and $|H|=|K|-1$ (Lemma 4.4).

If $G=H K$ is a Frobenius group, then by Proposition 2.1(a), there exist three possibilities:

(1) Both $|H|$ and $|K|$ are odd;

(2) $|H|$ is odd and $|K|$ is even;

(3) $|H|$ is even and $|K|$ is odd (necessarily $K$ is Abelian).

With one fell swoop, the next theorem dispenses with case (1):

Theorem 5.1 Let $G=H K$ be a Frobenius group of order less than $3 \cdot 7^{3}$ with $|H|$ and $|K|$ odd. Then $K$ is Abelian, and $G$ admits no GFR.

Proof Suppose that $G$ is of odd order $<3 \cdot 7^{3}$ but that $K$ is not Abelian. Since $K$ is nilpotent, for some odd prime $p$, the Sylow $p$-subgroup $\operatorname{Syl}_{p}(K)$ is not Abelian. Hence, $|K|$ is divisible by $p^{e}=\left|\operatorname{Syl}_{p}(K)\right|$ for some $e \geq 3$. Since $p^{e} \leq|K|=$ $|G| /|H| \leq 3 \cdot 7^{3} / 3=343$, we have either $p=3$ with $e \in\{3,4,5\}$ or $p=5$ with $e=3$. Since $\operatorname{Syl}_{p}(K)$ is not Abelian, $|H|$ divides $3-1,3^{2}-1$ or $5-1$ by Corollary 2.10. Since $|H|$ is odd, this is impossible. Thus, $K$ is Abelian and $G=H K$ does not have a GFR, again since $|H|$ is odd.

Remark 5.2 The bound of 1029 in Theorem 5.1 is, in fact, best possible. Using MAGMA, Marston Conder has found a GFR of a Frobenius group of order 1029. This group, presented abstractly in [30] as Exercise 12.6.23, has as kernel the nonAbelian group of order $7^{3}$ and exponent 7 . Conder's GFR on 343 vertices has valence 18. 
We next dispose of case (2), namely when $|K|$ even and $|H|$ is (necessarily) odd:

Theorem 5.3 Let $G=H K$ be a Frobenius group such that $|K|$ is even. Suppose $|G| \leq 300$ but $|G| \neq 192$. Then $K$ is Abelian and $G$ admits no GFR.

Proof Let $\left|\operatorname{Syl}_{2}(K)\right|=2^{e}$. Since $|H| \geq 3$ we have $e>1$; since $|G| \leq 300$, we have $2 \leq e \leq 6$. If $e=6$, then $|G|=192$. If $e=2,3$, or 5 , then $2^{e}-1$ is prime, and so $|H|=2^{e}-1$, making $\operatorname{Syl}_{2}(K)=\left(\mathbb{Z}_{2}\right)^{e}$. Since there is no odd cube less than $|K| / 2^{e} \leq 100 / 4=25$ and $K$ is nilpotent, this makes $K$ Abelian. Thus, when $e=2,3$, or 5 , there is no GFR of $G$ by Lemma 4.2 when $K \neq \operatorname{Syl}_{2}(K)$ and by Lemma 4.4 when $K=\operatorname{Syl}_{2}(K)$.

We are left with the case $e=4$.

Suppose that $K$ is non-Abelian. Then $K^{\prime}=\operatorname{Syl}_{2}(K)$ is also non-Abelian, since $K$ is nilpotent and $|K|$ is not divisible by an odd cube. Then $H K^{\prime}$ is a Frobenius group with a non-Abelian kernel $K^{\prime}$ of order 16 . Let $N$ be either the center or the commutator subgroup of $K^{\prime}$. Since $|H|$ divides both $|N|-1$ and $\left|K^{\prime} / N\right|-1$, we must have $|N|=4$ and $|H|=3$. Thus, both $N$ and $K^{\prime} / N$ are $\left(\mathbb{Z}_{2}\right)^{2}$. One may now simply run through a list of the nine non-Abelian groups of order 16 and verify that none has both its center and its commutator subgroup, as well as their quotients, equal to $\left(\mathbb{Z}_{2}\right)^{2}$. We conclude that there is no such Frobenius group when $K$ is non-Abelian.

If $K$ is Abelian but not an elementary Abelian 2-group, then by Lemma 4.2, $G$ has no GFR. If $K=\left(\mathbb{Z}_{2}\right)^{4}$ holds, then $|H|=3$ or 5 . (Clearly $|H| \neq 15$ by Lemma 4.4.) Thus, $H$ is cyclic, generated by an element $h$. Suppose that $\Gamma=\operatorname{Cay}(K, S)$ is a GFR of $G$. By Remark 4.6, we may assume that $\Gamma$ has valence $|S| \leq\lfloor(|K|-1) / 2\rfloor=7$.

If $H=C_{5}$, then $S$ consists of a single orbit of the form $\left(u_{1}, u_{2}, u_{3}, u_{4}, u_{1}+\right.$ $\left.u_{2}+u_{3}+u_{4}\right)$, since $H$ fixes the sum, namely $\mathbf{0}$, of all the elements in an orbit. But then there exist unwanted automorphisms of $K$ that permute the subset $\left\{u_{1}, \ldots u_{4}\right\}$. If $H=C_{3}$, then no single $H$-orbit generates $K$. Since $|S| \leq 7$, the set $S$ consists of exactly two orbits, which may be expressed as $\left(x, h(x), h^{2}(x)\right)$ and $\left(y, h(y), h^{2}(y)\right)$, where $x+h(x)+h^{2}(x)=y+h(y)+h^{2}(y)=\mathbf{0}$. The automorphism of $K$ that interchanges $x$ with $y$ and $h(x)$ with $h(y)$ is an unwanted group automorphism, and so $G$ admits no GFR.

Remark 5.4 When $|G|=192$, then clearly $|H|=3$ and $|K|=64$. If $K$ is Abelian but not an elementary Abelian 2-group, there is no GFR by Lemma 4.2. When $K=\left(\mathbb{Z}_{2}\right)^{6}$, there does exist one Frobenius group, but it has no GFR by Conder's computations with MAGMA. If $K$ is non-Abelian, then its center $N$ is nontrivial and so $|N|=4$ or 16, since 3 divides $|N|-1$. Since there exists no Frobenius group of order 16 whose kernel is non-Abelian, the possibilities for $N$ and $K / N$ are $\left(\mathbb{Z}_{2}\right)^{2},\left(\mathbb{Z}_{2}\right)^{4}$, and $\left(\mathbb{Z}_{4}\right)^{2}$. Again by MAGMA, there exists one Frobenius group with $N=\left(\mathbb{Z}_{2}\right)^{2}$ and $K / N=\left(\mathbb{Z}_{2}\right)^{4}$ that has a GFR; its valence is 15 .

For case (3), we have that $|H|$ is even and $|K|$ is odd (and necessarily Abelian). We make the following group-theoretical observation at the outset:

Proposition 5.5 Suppose that $A$ is an Abelian group, $H$ is a subgroup of $\operatorname{Aut}(A)$, and $c$ is a positive integer relatively prime to the exponent of $A$. Then scalar multiplication $x \mapsto c x$ permutes the orbits of $H$. 
Proof Since $A$ is Abelian and $c$ is relatively prime to the exponent of $A$, scalar multiplication by $c$ is an automorphism of $A$. If $h \in H$ and $y=h(x)$, then $h(c x)=c h(x)=c y$. Thus, if $x$ and $y$ belong to the same $H$-orbit, then so do $c x$ and $c y$.

Theorem 5.6 Suppose that $G=H K$ is a Frobenius group such that $|G| \leq 300$ with $|H|$ even and $|H| \neq|K|-1$. Then $G$ admits a GFR except in exactly the following cases:

(a) $K=\left(\mathbb{Z}_{3}\right)^{2}$;

(b) $K=\left(\mathbb{Z}_{3}\right)^{3}$;

(c) $K=\left(\mathbb{Z}_{5}\right)^{2}$ with $H$ generated by a scalar matrix of order 4 ;

(d) $K=\left(\mathbb{Z}_{5}\right)^{2}$ with $|H|>4$;

(e) $K=\left(\mathbb{Z}_{7}\right)^{2}$ with $H$ generated by a scalar matrix of order 6 .

Proof Since $H$ is even, $K$ is Abelian, $|K|$ is odd, and $\alpha$ is the sole involution in $H$. If $K$ is cyclic, then $G$ admits a GFR by Theorem 4.9. If $|H|=2$, then by Theorem 4.3, $H K$ has a GFR except when $K=\left(\mathbb{Z}_{3}\right)^{2}$ or $\left(\mathbb{Z}_{3}\right)^{3}$. In fact, since $|H|$ must be even and less than $|K|-1$, there is never any GFR at all when $K=\left(\mathbb{Z}_{3}\right)^{3}$ (completing Case (b)).

We henceforth assume $|H| \geq 4$, and so $|K| \leq 75$ and $\left(\mathbb{Z}_{p}\right)^{2} \leq K$ for some $p=3$, 5, or 7 . When $|K|=5^{2} \cdot 3$, then $|H|$ divides $3-1$, and when $|K|=3^{2} \cdot 7$, then $|H|$ divides both $9-1$ and $7-1$, again implying $|H|=2$. We conclude that the only possibilities for $K$ remaining to be considered are

$$
\left(\mathbb{Z}_{3}\right)^{2}, \quad\left(\mathbb{Z}_{3}\right)^{2} \times \mathbb{Z}_{5}, \quad\left(\mathbb{Z}_{5}\right)^{2}, \quad \text { and } \quad\left(\mathbb{Z}_{7}\right)^{2}
$$

$K=\left(\mathbb{Z}_{3}\right)^{2}$

The valence of any Cayley graph for $\mathbb{Z}_{3}^{2}$ is at least 4 , and hence by Remark 4.6, any GFR for $H K$ has valence exactly 4 . Thus, $S=x, y,-x,-y$ which has an extra group automorphism interchanging $x, y$. Alternatively, by [20] (Lemma 2.4), the least size of a vertex stabilizer of a Cayley graph of $\left(\mathbb{Z}_{3}\right)^{2}$ is 8 . But if $|H|=8$, there is no GFR by Lemma 4.4. (This completes Case (a).)

$K=\left(\mathbb{Z}_{3}\right)^{2} \times \mathbb{Z}_{5}$

Since $\mathbb{Z}_{5}$ is a characteristic subgroup of $K$, we must have that $|H|=4$ and $H$ is cyclic. Let the action of $H$ be given by $((a, b), c) \mapsto((-b, a), 2 c)$. If we set $x=((1,0), 1)$, then the orbit $H x$ generates $K$, and we may now invoke Theorem 4.12 to obtain a 4-valent GFR.

$K=\left(\mathbb{Z}_{5}\right)^{2}$

$\overline{\text { By Remark }} 4.6$, we may assume that the connection set $S$ of any GFR satisfies $|S| \leq 12$, and so $|H|=4,6,8$, or 12 .

If $|H|=4$, there exist two Frobenius groups $H\left(\mathbb{Z}_{5}\right)^{2}$ depending upon whether $H$ is generated by a scalar or a nonscalar matrix in $\operatorname{GL}(2,5)$, as described in Example 2.12. In the nonscalar case, we have a GFR of valence 8 by Theorem 4.13. In the scalar case, we note that the connection set contains at most three $H$-orbits. An orbit $H x$ is the 1-dimensional subspace spanned by an element $x$, which we denote $[x]$. Since a single orbit fails to generate $K$, any two vectors chosen from different $H$-orbits form 
an independent set. If $S=[x] \cup[y]$, the linear transformation interchanging $x$ and $y$ is unwanted. If $S$ consists of three $H$-orbits, then any vector $z$ in the third orbit can be written as $x+y$ for some vectors $x$ and $y$ in each of the other two orbits. Then the linear transformation interchanging $x$ and $y$ fixes $z$ and provides an unwanted automorphism. Hence, no GFR exists when $H$ is generated by a scalar matrix. (This completes case (c).)

If $|H|=6$, then $H=C_{6}$ since $H$ contains a unique involution. The only Frobenius group has $H$ generated by the matrix $\left(\begin{array}{cc}0 & -1 \\ 1 & 1\end{array}\right)$ (up to change of basis). The four $H$-orbits in $K$ are those of $(1,0),(2,0),(1,1)$, and $(2,2)$. Each is invariant under interchange of the first and second coordinates. Thus, there is always an unwanted group automorphism, and so $G$ has no GFR.

If $|H|=8$, then $S$ consists of a single $H$-orbit. Since $H$ contains a unique involution by Corollary $2.5, H$ is cyclic or quaternion and hence must also contain an element $g$ of order 4. Each $H$-orbit is a union of two orbits of $g$. If $g$ is represented by a scalar matrix, then by our analysis above for $|H|=4$ (with $H^{\prime}=\langle g\rangle$ in place of $H$ ), there is no GFR for $H^{\prime} K$. Hence, there is no GFR for $H K$ by Proposition 4.15. Otherwise, $g$ is represented (up to change of basis) by the nonscalar matrix $\left(\begin{array}{cc}0 & -1 \\ 1 & 0\end{array}\right)$. The orbits of $g$ are $O_{1}=((1,0),(0,1), \ldots), O_{2}=((1,1),(-1,1), \ldots)$, $O_{3}=((1,2),(-2,1), \ldots)$, and $O_{4}=((2,1),(-1,2), \ldots)$ and the scalar multiples $2 \mathrm{O}_{1}$ and $2 \mathrm{O}_{2}$. Since interchange of coordinates leaves invariant each of the orbits $\mathrm{O}_{1}, 2 \mathrm{O}_{1}, \mathrm{O}_{2}$, and $2 \mathrm{O}_{2}$ while interchanging $\mathrm{O}_{3}$ with $\mathrm{O}_{4}$, the connection set $\mathrm{S}$ of any GFR, being of size 8, must contain exactly one of $\mathrm{O}_{3}$ and $\mathrm{O}_{4}$. But that is impossible since $\mathrm{O}_{3}$ and $\mathrm{O}_{4}$ are each invariant under scalar multiplication and the other orbits are not, contradicting Proposition 5.5, applied to the $H$-orbits.

If $|H|=12$, then $H$ is cyclic or dicyclic, since $H$ contains a unique involution. Thus, $H$ contains an element $g$ of order 6 . It was shown in the nonscalar case of $|H|=6$ that interchanging $x$ with $y=h(x)$ is a group automorphism leaving all orbits of $g$ invariant. Since this automorphism fixes $x+y$, we can apply Proposition 4.15 to conclude there is no GFR (completing Case (d)).

$K=\left(\mathbb{Z}_{7}\right)^{2}$

Since $49|H| \leq 300$, we consider only $|H|=4$ and $|H|=6$, and in both cases, $H$ must be cyclic, say $H=\langle h\rangle$. If $h$ is represented by a nonscalar matrix, then there exists $x \in K$ such that $\{x, h(x)\}$ is linearly independent, and so $H x$ generates $K$. The hypotheses of Theorem 4.12 when $|H|=4$ and of Theorem 4.14 when $|H|=6$ are now satisfied. Hence, $G$ admits a GFR.

It remains only to show that no GFR exists when $H$ is generated by a scalar matrix. Since there is no scalar matrix in GL $(2,7)$ of order 4 , we assume that $H=C_{6}$. Thus, $K \backslash\{i d\}$ admits a partition into eight $H$-orbits. As we have shown in the previous case of scalar matrices for $K=\left(\mathbb{Z}_{5}\right)^{2}$, the connection set of a GFR must contain more than three, and hence exactly four $H$-orbits $[x],[y],[z]$, and $[w]$, and one may assume $z=x+y$. With respect to the basis $\{x, y\}$, consider the matrices $A=\left(\begin{array}{l}0-1 \\ 1-1\end{array}\right)$ and $B=\left(\begin{array}{ll}0 & 1 \\ 1 & 0\end{array}\right)$ in $\operatorname{GL}(2,7)$. Then $A^{3}=I$ and $A$ maps $x \mapsto y \mapsto-z \mapsto x$, while $B$ 
interchanges $x$ and $y$ and fixes $z$. Thus, both $A$ and $B$ represent group automorphisms not in $H$ (because they're nonscalar) that leave invariant the set $\{[x],[y],[z]\}$.

If $[w]=[x-y]$, then $B$ is an unwanted automorphism, since it fixes $[x-y]$ and hence fixes $S$. The orbit of $[x-y]$ under $A$ is $\{[x-y],[x+2 y],[-2 x-y)]\}$. Each of the remaining two $H$-orbits must be fixed by $A$ since $A$ has order 3 . If $[w]$ is either of these two orbits, then $A$ is an unwanted automorphism. Finally, if $[w]=A[x-y]$, then $A B A^{-1}$ leaves $[w]$ invariant. Since $A$ and $B$ leave the set $\{[x],[y],[z]\}$ invariant, $A B A^{-1}$ is an unwanted automorphism. If $[w]=-A^{2}[x-y]$, then $A^{2} B A^{-2}$ leaves $[w]$ invariant and hence is an unwanted automorphism. We conclude that no GFR of $H K$ exists (and finally that Case (e) is excluded).

Remark 5.7 In the proofs above of the nonexistence of a GFR when $K=\left(\mathbb{Z}_{5}\right)^{2}$ and $|H|=8$ or 12 , we implicitly assumed the existence of such a Frobenius group. For $|H|=8$, we stated merely that $H$ is either cyclic or quaternion. In both instances, the Frobenius groups indeed exist. The quaternion group is generated by the matrices $A=\left(\begin{array}{ll}0 & 2 \\ 2 & 0\end{array}\right)$ and $B=\left(\begin{array}{cc}2 & 0 \\ 0 & -2\end{array}\right)$. One easily checks that $A^{2}=B^{2}=(A B)^{2}=\alpha$. When $H$ is cyclic, we use $C=\left(\begin{array}{ll}0 & 2 \\ 1 & 0\end{array}\right)$.

Similarly, when $|H|=12$ holds, the only noncyclic possibility for $H$ is the dicyclic group generated by $A=\left(\begin{array}{cc}0 & -1 \\ 1 & 1\end{array}\right)$ and $B=\left(\begin{array}{ll}0 & 2 \\ 2 & 0\end{array}\right)$. As we know, $A^{6}=I$. One easily checks that $B^{4}=I$ and $B^{-1} A B=A^{-1}$. For $H=C_{12}$, use $2 A^{2}$, which has order 12 since $A^{2}$ has order 3 .

Summary The only Frobenius groups $G=H K$ with $|G| \leq 300$ and $|G| \neq 192$ that admit a GFR are those guaranteed by Theorems 4.3, 4.9, 4.12, 4.13, and 4.14, for all of which $|H|$ is even. For $|G|=192$, computation with MAGMA shows that there is exactly one Frobenius group having a GFR; it has $|H|=3$ and $K$ non-Abelian.

Table 1 summarizes the GFR status of all Frobenius groups $G=H K$ of order $|G| \leq 300,|G| \neq 192$. Omitted are those Frobenius groups for which GFRs are precluded by Lemmas 4.2 and 4.4 and Theorems 5.1 and 5.3, and we omit the many GFRs that are assured by Theorems 4.3 and 4.9 .

\section{Questions}

Various questions arise naturally from our computations in Sect. 5, where much of the work is devoted to the cases with $K=\left(\mathbb{Z}_{p}\right)^{2}$ for $p=5$ and 7 . The subcases where $H$ is generated by a scalar matrix lead to Frobenius groups without a GFR for both of these small values of $p$. Is this merely an example of "low-level noise?" More specifically, we pose the following.

Question 6.1 If $K=\left(\mathbb{Z}_{p}\right)^{n}$ for an odd prime $p$ and $H$ is generated by a scalar matrix of even order, does the Frobenius group $G=H K$ admit a GFR whenever $p$ is sufficiently large compared to $n$ ? 
Table 1 GFR status of sporadic Frobenius groups of order $\leq 300$ (see comment about omitted groups)

\begin{tabular}{lllll}
\hline$|G|$ & $H$ & $K$ & Valence & Relevant theorem $(\mathrm{s})$ \\
\hline 36 & $C_{4}$ & $\left(\mathbb{Z}_{3}\right)^{2}$ & No GFR & 5.6 \\
48 & $C_{3}$ & $\left(\mathbb{Z}_{2}\right)^{4}$ & No GFR & 5.3 \\
80 & $C_{5}$ & $\left(\mathbb{Z}_{2}\right)^{4}$ & No GFR & 5.3 \\
100 & $C_{4}$, nonscalar & $\left(\mathbb{Z}_{5}\right)^{2}$ & 8 & 4.13 \\
100 & $C_{4}$, scalar & $\left(\mathbb{Z}_{5}\right)^{2}$ & No GFR & 5.6 \\
150 & $C_{6}$ & $\left(\mathbb{Z}_{5}\right)^{2}$ & No GFR & 5.6 \\
180 & $C_{4}$ & $\left(\mathbb{Z}_{3}\right)^{2} \times \mathbb{Z}_{5}$ & 4 & 4.12 and 5.6 \\
196 & $C_{4}$, nonscalar & $\left(\mathbb{Z}_{7}\right)^{2}$ & 8 & 4.13 \\
200 & $C_{8}$ & $\left(\mathbb{Z}_{5}\right)^{2}$ & No GFR & 5.6 \\
200 & $Q$ & $\left(\mathbb{Z}_{5}\right)^{2}$ & No GFR & 5.6 \\
294 & $C_{6}$, nonscalar & $\left(\mathbb{Z}_{7}\right)^{2}$ & 12 & 4.14 \\
294 & $C_{6}$, scalar & $\left(\mathbb{Z}_{7}\right)^{2}$ & No GFR & 5.6 \\
300 & $C_{12}$ & $\left(\mathbb{Z}_{5}\right)^{2}$ & No GFR & 5.6 \\
300 & Dicyclic & $\left(\mathbb{Z}_{5}\right)^{2}$ & No GFR & 5.6 \\
\hline
\end{tabular}

It was shown in Theorem 5.6 that the Frobenius group $C_{6}\left(\mathbb{Z}_{5}\right)^{2}$ admits no GFR. This may appear to be a similar case of low-level noise, but this time it is not. In a sequel [6] to the present work, it is shown that:

Theorem 6.2 Suppose that $K=\left(\mathbb{Z}_{p}\right)^{2}$ and $H=C_{p+1}$, where $p \equiv 1(\bmod 4)$ is prime. Then there is an involution in $\operatorname{GL}(2, p)$ not in $H$ that leaves all orbits of $H$ invariant. In particular, there is no GFR for $G=H K$.

It was mentioned early on that all but finitely many of the groups that do not admit GRRs belong to one of only two infinite classes (namely Abelian groups that are not elementary Abelian 2-groups and generalized dicyclic groups). Do Frobenius groups not admitting GFRs demonstrate similar behavior? Specifically, one might ask:

Question 6.3 Do all but finitely many Frobenius groups that fail to have a GFR fall into a small number of infinite families?

For the GRR problem, beyond low-level noise, the groups in those two exceptional families almost admit GRRs (see [20,25]). The only problem is the ever-presence of automorphisms that either fix or reverse the direction of each arc of their Cayley graphs. An exception are Cayley graphs of those generalized dicyclic groups of the form $Q \times\left(\mathbb{Z}_{2}\right)^{n}$, whose vertex stabilizers are bounded by 16 (see [20]). One can define for a connection set $S$, not necessarily closed under inverses, a directed Cayley graph, i.e., where there is an arc from $x$ to $y$ if and only if $y=x s$ for some $s \in S$. The DRR problem is to determine all groups that act regularly on a directed graph (as automorphisms that respect directions). In this case, all but five groups have a DRR (see L. Babai [1]). One can pose the same problem for GFRs. We note that when $|H|$ 
is even, there is always the involution $\alpha \in H$ which reverses edge directions, and so if we want directed graph automorphisms we must restrict our attention to odd $|H|$.

Question 6.4 (The DFR problem) Given a Frobenius group $G=H K$ with $|H|$ odd, when does there exist a directed graph representation (DFR) for $G$, namely a directed graph whose automorphism group acting on the vertices is the same as $G$ ?

We wonder whether smaller Frobenius groups that admit a GFR can "beget" larger ones that also do. In the course of solving the GRR problem, it was found that, barring low-level noise, if $G$ and $G^{\prime}$ are groups that admit a GRR then so is $G \times G^{\prime}$, and so are most cyclic extensions of $G$. This worked particularly well in constructing GRRs of solvable groups. While products are precluded as a means of "growing" Frobenius groups (cf. Theorem 3.3), we ask whether the process of Lemma 2.9 can be reversed in the following sense:

Question 6.5 Suppose that $G=H K$ is a Frobenius groups and $N$ is a characteristic subgroup of $K$. If both $H N$ and $H(K / N)$ admit GFRs, must then $G$ itself admit a GFR?

We note that the converse, namely if $G=H K$ has a GFR, then so do $H N$ and $H(K / N)$, is false. In Theorem 5.6 we saw that if $K=\mathbb{Z}_{3}^{2} \times \mathbb{Z}_{5}$ and $H=C_{4}$, then $H K$ has a GFR. However, if $N=\mathbb{Z}_{5}$, then neither $H N$ nor $H(K / N)$ admits a GFR.

One may approach these questions by first solving two less ambitious problems:

Problem 1 Find all GFRs when $K=\left(\mathbb{Z}_{p}\right)^{2}$ and $H$ is cyclic.

Problem 2 Find all GFRs when $K=\left(\mathbb{Z}_{2}\right)^{n}$ and $H$ is cyclic.

Finally, we propose an ambitious conjecture. Although Theorem 6.2 gives a family of Frobenius groups not having a GFR, the size of $H$ is not bounded. Suppose we fix $H$ ? For the GRR problem, in effect, we have $|H|=1$ and we conclude that except for two families, all groups have a GRR except for low-level noise. The appropriate analogy for the GFR problem is to eliminate the ceiling of 300 in Theorem 5.3 and pose the following:

Conjecture (The GFR Conjecture) All but finitely many Frobenius groups $H \mathrm{~K}$ with a given complement $H$ have a GFR, except when $|H|$ is odd and $K$ is Abelian but not an elementary Abelian 2-group.

Acknowledgements Parts of this work were contained in the first author's Ph.D. dissertation [10] at Syracuse University, written under the supervision of the third author. Please see the last paragraph of Introduction concerning the contributions of Marston Conder. Mark Watkins and Thomas Tucker were partially supported by collaborative research grants from the Simons Foundation (\#209803 to Watkins and \#317689 to Tucker).

\section{References}

1. Babai, L.: Finite digraphs with given regular automorphism groups. Per. Math. Hungar. 11, 257-270 (1980) 
2. Babai, L., Godsil, C.D.: On the automorphism groups of almost all Cayley graphs. Eur. J. Combin. 3, 9-15 (1982)

3. Bhoumik, S., Dobson, E., Morris, J.: On the automorphism groups of almost all circulant graphs and digraphs. Ars Math. Contemp. 7, 487-506 (2014)

4. Bosma, W., Cannon, J., Playoust, C.: The Magma algebra system I: the user language. J. Symb. Comput. 24, 235-265 (1997)

5. Collins, M.J.: Some infinite Frobenius groups. J. Algebra 131, 161-165 (1990)

6. Conder., M.D.E., Spiga, P., Tucker, T.W.: Exceptions for the GFR Problem (in progress)

7. Dixon, J.D., Mortimer, B.: Permutation Groups. Springer, New York (1996)

8. Dobson, E.: Asymptotic automorphism groups of Cayley digraphs and graphs of abelian groups of prime-power order. Ars Math. Contemp. 3, 200-213 (2010)

9. Dobson, E., Spiga, P., Verret, G.: Cayley graphs on Abelian groups. Combinatorica 36, 371-393 (2016)

10. Doyle, J.K.: Graphical Frobenius Representations of Abstract Groups, Ph.D. thesis, Syracuse University (1976)

11. Frobenius, F.G.: Über auflösbare Gruppen IV, Berliner Sitz. pp. 1216-1230 (1901)

12. Godsil, C.D.: GRRs for nonsolvable groups. Colloq. Math. Soc. János Bolyai 25, 221-239 (1981)

13. Godsil, C.D.: On the full automorphism group of a graph. Combinatorica 1, 243-256 (1981)

14. Hetzel, D.: Über reguläre graphische Darstellung von auflösbaren Gruppen, Diplomarbeit Fachbereich Mathematik, Technische Universität Berlin (1977)

15. Imrich, W.: Graphs with transitive Abelian automorphism groups. In: Combinatorial Theory and Its Applications, Colloq. Math. Soc. János Bolyai 4, pp. 651-656. Balatonfúred, Hungary (1969)

16. Imrich, W.: Graphical Regular Representations of Groups of Odd Order, Colloq. Math. Soc. János Bolyai 18, Combinatorics, Keszthely, Hungary, pp. 611-621 (1976)

17. Imrich, W., Izbicki, H.: Associative products of graphs. Monatsh. Math. 80, 277-281 (1975)

18. Imrich, W., Klavžar, S.: Product Graphs. Structure and Recognition, Wiley, New York (2000)

19. Imrich, W., Watkins, M.E.: On graphical regular representations of cyclic extensions of groups. Pac. J. Math. 54, 1-17 (1974)

20. Imrich, W., Watkins, M.E.: On automorphism groups of Cayley graphs. Per. Math. Hungar. 7, 243-258 (1976)

21. Kovács, I.: Classifying arc-transitive circulants. J. Algebr. Combin. 20, 353-358 (2004)

22. Li, C.H.: Permutation groups with a cyclic regular subgroup and arc transitive circulants. J. Algebr. Combin. 21, 131-136 (2005)

23. Morris, J., Spiga, P.: Every finite non-solvable group admits an oriented regular representation. J. Combin. Theory Ser. B 126, 198-234 (2017)

24. Morris, J., Spiga, P., Verret, G.: Automorphisms of Cayley graphs on generalised dicyclic groups. Eur. J. Combin. 43, 68-81 (2015)

25. Morris, J., Tymburski, J.: Most rigid representations and Cayley index. Ars Math. Contemp. (to appear)

26. Nowitz, L.A., Watkins, M.E.: Graphical regular representations of non-abelian groups I, II. Canad. J. Math. 24(993-1008), 1009-1018 (1972)

27. Passman, D.S.: Permutation Groups. Benjamin, New York (1968)

28. Sabidussi, G.: On a class of fixed-point free graphs. Proc. Am. Math. Soc. 9, 800-804 (1958)

29. Sabidussi, G.: Vertex-transitive graphs. Monatsh. Math. 68, 426-438 (1964)

30. Scott, W.R.: Group Theory. Prentice-Hall, Englewood Cliffs (1964)

31. Thompson, J.G.: Finite groups with fixed-point-free automorphisms of prime order. Proc. Nat. Acad. Sci. USA 45, 578-581 (1959)

32. Watkins, M.E.: On the action of non-Abelian groups on graphs. J. Combin. Theory 11, 95-104 (1971)

33. Watkins, M.E.: On Graphical regular representations of $C_{n} \times Q$. Graph Theory and Its Applications. Conference at Western Michigan University, pp. 305-311. Springer, Berlin (1972)

34. Watkins, M.E.: Graphical regular representations of alternating, symmetric, and miscellaneous small groups. Aequat. Math. 11, 1-17 (1974)

35. Watkins, M.E.: The state of the GRR problem. In: Fiedler, M. (ed.) Recent Advances in Graph Theory, Proceedings of the Second Czechoslovak Conference on Graph Theory, pp. 517-522. Prague (1974)

36. Watkins, M.E.: Graphical regular representations of free products of groups. J. Combin. Theory Ser. B 21, 47-66 (1976)

37. Watkins, M.E.: Infinite Graphical Frobenius Representations (submitted)

38. Xu, M.Y.: Automorphism groups and isomorphisms of Cayley digraphs. Discrete Math. 182, 309-319 (1998) 